\title{
EDITORIAL
}

\section{Idiopathic pulmonary fibrosis: guidelines for diagnosis and clinical management have advanced from consensus-based in 2000 to evidence-based in 2011}

\author{
G. Raghu
}

I diopathic pulmonary fibrosis (IPF) is increasingly recognised as a well-known clinical and highly complex entity associated with poor prognosis and a median survival of 3-5 yrs after the diagnosis is made. The importance of the need for an accurate diagnosis and appropriate clinical management is evident. In this regard, the first statement/guidelines on the clinical management of IPF, jointly produced by the American Thoracic Society (ATS) and the European Respiratory Society (ERS), provided recommendations that were essentially based on the consensus of the opinions of a few experts, as the evidence available at that time was minimal [1]. In 2000, when the statement was published, the common practice was to develop guidelines based upon available research using a consensus-based approach to formulate recommendations for management. Thus, the recommendations suggested in the first statement were based on a consensus of opinions more than the evidence. Over the years, this approach has evolved and most guideline developers are now using a more robust methodological approach; the umbrella term is "evidencebased medicine", which includes grading of the evidence using a standard system (e.g. Grading of Recommendations Assessment, Development and Evaluation (GRADE)).

As the research available for clinical management of IPF was sketchy when the first guideline for the management of IPF was developed, the limitations associated with the recommendations suggested in the consensus-based statement were acknowledged. Nevertheless, the ATS/ERS guideline identified clinical awareness of IPF as a distinct entity, provided major and minor clinical criteria for diagnosis of IPF, and suggested recommendations for treatment, monitoring and assessment of response to treatment. This was very useful to the pulmonary community at large; clinicians and patients worldwide began to have a better understanding of the poor/ fatal prognosis associated with IPF, and abundant clinical studies emerged. Through use of the consensus-based statement/guideline for IPF, significant progress has been made regarding the clinical management and course for patients with IPF and a very large body of evidence has been accumulated over the last decade.

CORRESPONDENCE: G. Raghu, Division of Pulmonary and Critical Care Medicine, UW Medical Centre, Seattle, WA, USA. E-mail: graghu@u.washington.edu
The absolute need for a new guideline, this time evidencebased, was increasingly evident. A large panel of international clinical experts in the field of interstitial lung diseases (ILD), idiopathic interstitial pneumonia (IIP) and IPF, who represent the ATS, the ERS, the Japanese Respiratory Society (JRS) and the Asociación Latinoamericana de Tórax (ALAT), have reviewed the extensive literature published to date that is pertinent to the clinical management of IPF. This has resulted in the recent publication of guidelines for the accurate diagnosis of IPF and recommendations for its managements [2].

The recommendations for diagnosis and treatment interventions in this new, ATS/ERS/JRS/ALAT guideline are, for the very first time, evidence-based and eliminate the bias based on expert opinions and ongoing practices in the management of IPF [2]. The essence of evidence-based guidelines is to incorporate appropriate clinical information into patient care; if the evidence indicates more harm than benefit from intervention (and this is particularly the case for recommendations against specific interventions used frequently/routinely in current practice), appropriate changes need to be made in current practice. The quality of the available evidence was determined according to the ATS GRADE criteria [3], and the strength of the recommendations was determined by a vote amongst committee members. These votes followed discussion and consideration of the balance of potential desirable (i.e. benefits) and undesirable consequences (i.e. risks, burden, costs) of the intervention, the quality of evidence and the preferences of patients with typical features of IPF.

The methodology used in developing the new guidelines was therefore scientifically robust and in keeping with current approaches to "evidence-based medicine". In addition, there was transparency in the process that was followed by the committee members, the disclosure of all potential conflicts of interest, and in the results of the votes. Thus, the clinician confronted with a patient with typical IPF is, for the very first time, empowered to make an independent and the most appropriate decision for the patient using the evidence-based guidelines for clinical management of IPF.

\section{NEW DEFINITION AND NEW DIAGNOSTIC CRITERIA FOR IPF}

The definition of IPF has been refined as a progressive fibrosing interstitial pneumonia of unknown cause, occurring in adults, limited to the lungs and associated with the 
histopathological and/or radiological pattern of usual interstitial pneumonia (UIP).

The new guidelines raise the need to recognise the specific pattern of UIP, as diagnosis of IPF requires the presence of UIP pattern on high-resolution computed tomography (HRCT) images of the lungs and/or in the surgical lung biopsy (SLB) specimens obtained from patients in the appropriate clinical setting. (The typical clinical setting for IPF is the adult (commonly a male of $>60$ yrs of age) without collagen vascular disease and exclusion of causes known to cause and/or associated with ILD.)

The guidelines clearly state the precise HRCT features that meet the criteria for "UIP", "possible UIP" and "inconsistent with UIP" patterns. In the appropriate clinical setting, the presence of the described criteria for the pattern of UIP on HRCT images is sufficient to make an accurate diagnosis of IPF. In patients demonstrating features that meet the criteria for "possible UIP" and/or "inconsistent with UIP" patterns on HRCT images, the histopathological features of the SLB are required to make an accurate diagnosis of IPF. Precise histopathological features that meet the criteria for "UIP", "probable UIP", "possible UIP" and "not UIP" patterns are also clearly stated in the new guidelines. For patients whose HRCT and/or histopathological features in SLB specimens do not meet the specified criteria for the UIP patterns described (i.e. patients who meet the criteria for "possible UIP" and "inconsistent with UIP" patterns on HRCT, and patients whose histopathological features meet the specified criteria for "probable UIP", "possible UIP" and "not UIP" patterns), specific combinations of the HRCT and histopathology patterns in SLB are also given to increase the likelihood of accurate diagnosis of IPF.

Whilst the guideline criteria for HRCT images of the lungs and for histopathology of the SLB specimens will greatly enhance the clinician's ability to make an accurate diagnosis of IPF, multidisciplinary discussions (MDD) between pulmonologists, radiologists and pathologists experienced in the diagnosis of ILD will further increase the likelihood of accurate diagnosis. When MDD is not available/feasible in local practices, MDD in regional centres known to have expertise in the evaluation and management of ILD should be encouraged in order to make an accurate diagnosis, particularly when combinations of HRCT and histopathology are other than the definitive, UIP patterns.

In essence, the recognition of precise UIP patterns on HRCT and histopathology in the the appropriate clinical setting for the diagnosis of IPF is emphasised in the new guidelines. Exclusion of known causes of ILD relies upon a thorough history elicitation and clinical examination to exclude attributable domestic and occupational exposures, collagen vascular disease (including serology) and drug toxicity. The major and minor criteria established by the previous panel of IPF experts and proposed in the 2000 ATS/ERS IPF statement [1] are no longer required. Patients may have normal pulmonary function tests and yet have IPF. In the appropriate clinical setting, transbronchial lung biopsy or bronchoalveolar lavage cellular analysis are no longer required to make a diagnosis of IPF in patients manifesting HRCT features of UIP.

\section{TREATMENT RECOMMENDATIONS FOR PATIENTS WITH IPF}

It is important that both the clinician and the patient understand that the recommendations for treatment in the new guidelines were made following very careful and thorough review of all available evidence published before May 30, 2010. The quality of evidence was graded in accordance with the ATS statement [3], and the process was reviewed and defined with the committee members during a face-to face meeting before the vote took place. The strength of the recommendations provided was thus determined by the vote (as previously stated). The significance and implication of the recommendations for the patient are noted in table 1; the published guidelines elaborate on the significance of the recommendations for clinicians, patients and policy makers [2].

In essence, evidence published to date has revealed no proven pharmacological therapy for IPF. However, potential benefits

\section{TABLE 1 Evidence-based treatment recommendations}

\section{Strong yes}

Implication for the patient: most patients would want the following treatment intervention and only a small proportion would not.

1) Long-term oxygen therapy in patients with IPF demonstrating

clinically significant resting hypoxaemia

2) Lung transplantation in appropriate patients

\section{Weak yes}

Implication for the patient: a majority of patients would want the

following treatment intervention, but many would not. Not using them

may be a reasonable choice in a minority.

1) Corticosteroids for acute exacerbation of IPF

2) Treatment of asymptomatic gastro-oesophageal reflux

3) Pulmonary rehabilitation

Strong no

Implication for the patient: most patients would not want the

following treatment intervention and only a small

proportion would.

1) Monotherapy with corticosteroids

2) Colchicine

3) Cyclosporine $A$

4) Combined corticosteroid and immune modulator therapy

5) Interferon- $\gamma$

6) Bosentan

7) Etanercept

\section{Weak no}

Implication for the patient: the majority of patients would not want the following treatment intervention, but many would, i.e. the following treatment interventions should not be used in the majority of patients with IPF, but may be a reasonable choice in a minority.

1) Combined prednisone, azathioprine and NAC

2) Monotherapy with NAC

3) Anticoagulation

4) Pirfenidone

5) Pulmonary hypertension associated with IPF

6) Mechanical ventilation in patients with respiratory failure due to IPF

"Yes" is "for" the use of the specific treatment; "No" is "against" the use of the specific treatment. IPF: idiopathic pulmonary fibrosis; NAC: $\mathrm{N}$-acetyl-cysteine. Data from [2]. 
from some pharmacological agents were suggested in a few studies and the recommendations for treatment with such agents for IPF are listed in "Weak no" of table 1. Recognising that the well-informed patient may strongly desire pharmacological treatment for IPF, the committee suggested that the choice of treatment agent may be made from therapeutic interventions that received a weak recommendation against their use, i.e. a weak no.

It must be noted that the regulatory agencies that review applications seeking approval for the use of specific pharmaceutical agents for IPF treatment make decisions of approval according to the set policies and procedures of the individual agencies. The treatment recommendations made by the IPF committee (representing the ATS, ERS, JRS and ALAT) as evidence-based guidelines for the management of IPF are based on the methodology outlined in the guideline.

\section{IPF DISEASE PROGRESSION AND MONITORING DISEASE COURSE}

The new guideline also describes the natural course of patients with IPF [2]. While patients are generally known to progress steadily and slowly over a few years, this may vary: some patients follow a more stable course, whereas a subset may rapidly decline, and yet another subset may manifest acute respiratory decline (acute exacerbation of IPF) despite being stable for extended periods of time.

While the evidence to date allowed the IPF committee to make specific diagnosis and therapy recommendations as evidencebased guidelines, it did not allow the committee to make specific recommendations in accordance with the GRADE methodology for monitoring clinical course and progressive disease. Progressive dyspnoea, 10\% sustained decrease from baseline measurements in absolute forced vital capacity (FVC), $15 \%$ sustained decrease in absolute diffusing capacity of the lung for carbon monoxide ( $\mathrm{L}, \mathrm{CO}$; corrected for haemoglobin), progression of fibrosis patterns on HRCT images from baseline, manifestation of acute exacerbation, and death from respiratory failure in the absence of another identifiable cause, are considered consistent with progressive disease. Whilst the optimal time interval to measure FVC and DL,CO has not been determined, the new guidelines suggest routine monitoring at 3-6-month intervals and a flexible approach to earlier measurement for patients with progressive dyspnoea. It is recommended that monitoring for determination of the need for supplemental oxygen, using pulse oximetry at rest and exertion/walk tests, is performed at 3-6-month follow-up intervals, with careful attention to pulse oximetry tracing and poor signals due to poor peripheral circulation. The guidelines also alert clinicians to the need to monitor for complications during follow-up based on a high index of clinical suspicion (e.g. pulmonary embolus, deep venous thrombosus), and to be aware of comorbidities (e.g. emphysema, pulmonary hypertension) at the time of the diagnosis of IPF and/or during followup and monitoring disease course. Given that emphysema and IPF are characterised by destruction of pulmonary parenchyma due to independent risk factors and different pathogenesis, patients with combined emphysema and IPF may be sicker than patients manifesting one of these lung diseases. However, it is unclear whether patients manifesting combined emphysemaIPF, in contrast to patients manifesting IPF or emphysema alone, represent a distinct clinical phenotype/syndrome with a poorer prognosis and have unique genetic predisposition factors to manifest both of these entities.

\section{CURRENT STATUS FOR CLINICAL MANAGEMENT OF PATIENTS DIAGNOSED WITH IPF BASED ON EVIDENCE TO DATE}

There is no known medical therapy proven to have enhanced survival and improved outcomes for patients with IPF. Physicians are required to spend adequate time with patients to discuss their values, preferences and prognosis at the time of the diagnosis and when confronted with the patient with IPF. Patients at increased risk of mortality should be considered for lung transplantation. Features/factors and measurements at baseline and assessed longitudinally during follow-up that are associated with increased mortality are given in the new guidelines. Appropriate patients should be evaluated and listed for lung transplantation in a timely manner; these include patients manifesting features/factors associated with high risk for mortality at diagnosis itself. Nonpharmacolocgical (oxygen supplementation, pulmonary rehabilitation) and pharmacological treatment interventions should be based on the recommendations (table 1); pharmacological treatment should be limited to the minority of patients who are willing to accept possible adverse consequences even if expected benefits are small. This is especially so for therapies recommended as "weak no" and these should be discussed with the patient and tailored to their individual values and preferences. All patients should be monitored for disease progression at 4-6 months or sooner as clinically indicated and identify potential complications. Selected patients manifesting disease progression during follow-up must undergo evaluation for consideration of lung transplantation. Corticosteroids are an appropriate treatment option for acute exacerbation. Mechanical ventilation is not recommended in the majority of patients with respiratory failure due to the progression of their disease. Symptom control (palliative care) focuses on reducing symptoms (e.g. cough, dyspnoea) providing comfort to patients, rather than treating disease. Advance directives must be discussed in the ambulatory setting. Acknowledging that there is no known effective pharmacological intervention to date, patients should be made aware of available clinical trials for possible enrolment at all stages of their disease course [4].

It must be recognised that the recommendations provided in the new evidence-based guideline are intended for patients manifesting typical clinical features of IPF. The recommendations may not therefore be acceptable to the individual patient and to all patients, and a minority of patients may want to accept the possible adverse effects and very small potential benefits associated with an intervention. Thus, it is imperative for the clinician to spend much-needed time with the patient and/or caregiver to discuss the treatment recommendations in-depth and to make the most appropriate decisions tailored to individual patient's needs and preferences. This is especially the case for the "weak" recommendations.

\section{FUTURE DIRECTIONS}

Evidence to date clearly warrants the continued need for welldesigned clinical trials to determine safe and effective 
treatment interventions that improve outcomes, quality of life, and survival [4]. It is hoped that concerted efforts and discussion among clinical investigators and regulatory agencies will lead to the most appropriate choice of clinically significant and meaningful end-points in clinical trials assessing treatment response in IPF. There are several clinical trials and studies in IPF that are underway (ClinicalTrials.gov) and others will hopefully be designed to utilise the new definitions, diagnostic criteria and evolved knowledge in the clinical management of IPF as posited in the new guidelines [2]. It is hoped that the results from ongoing and new clinical trials will soon determine an effective treatment for patients with IPF. The just-published new guidelines will need to be regularly updated as pertinent new evidence unfolds.

There is an absolute need for continued studies to understand and predict IPF natural history, identify biomarkers and predictors of disease progression and prognosis, and detect disease in preclinical and early stages. Genetic studies and strategies to prevent and regenerate lung tissue, lessons and knowledge from cancer biology [5], stem cell transplant and gene therapy all hold promise in ultimately curing IPF. This can only be accomplished through continued education of patients [6] and physicians, as well as through their willingness to participate in studies; co-operative, collective efforts and collaboration with clinicians, basic scientists, clinical investigators, sponsors and patient advocacy groups (e.g. the Pulmonary Fibrosis Foundation in Chicago, IL, USA, and the Coalition for Pulmonary Fibrosis, Culver City, CA, USA) are needed. It is also hoped that recently initiated networks, such as IPFnet in the USA (www.IPFnet.org) and the European IPF Network (www.pulmonary-fibrosis.net) in Europe [7-9], as well as the increasing network of clinical sites that have participated in IPF clinical trials worldwide, will be able to continue working together with the continued support of sponsors, and provide much-needed evidence documenting the improvement of standards of care and outcomes for patients with IPF in the near future.

\section{STATEMENT OF INTEREST}

A statement of interest for G. Raghu can be found at www.erj. ersjournals.com/site/misc/statements.xhtml

\section{ACKNOWLEDGEMENTS}

I am indebted to the following experts who served as committee members with me in developing the new evidence-based guidelines for the management of idiopathic pulmonary fibrosis (IPF): J. Ancochea (Universidad Auonoma de Madrid, Madrid, Spain), J. Behr (University of Munich, Munich, Germany), D. Bouros (Democritus University of Thrace, Athens, Greece), K. Brown (National Jewish Health, Denver, CO, USA), C. Carvalho (University of São Paulo, São Paulo, Brazil), T. Colby (Mayo Clinic, Scottsdale, AZ, USA), H. Collard (University of California, San Francisco, CA, USA), J-F. Cordier (Louis Pradel Heart and Lung Hospital, Lyon, France), U. Costabel (University of Essen,
Essen, Germany), R. du Bois (Imperial College, London, UK), M. Ebina (Tohoku University, Sendai, Japan) J. Egan (University College Dublin, Dublin, Ireland), K. Flaherty (University of Michigan, Ann Arbor, MI, USA), D. Hansell (Royal Brompton Hospital, London, UK), T. Johkoh (Kinki Central Hospital, Itami, Japan), D.S. Kim (University of Ulsan, Seoul, Korea), T. King Jr (University of California), Y. Kondoh (Tosei General Hospital, Seto, Japan), J. Lasky (Tulane University, New Orleans, LA, USA), D. Lynch (National Jewish Health), F. Martinez (University of Michigan), N. Muller (University of British Colombia, Vancouver, Canada), J. Myers (University of Michigan), A. Nicholson (Royal Brompton Hospital), L. Richeldi (University of Modena and Reggio, Emilia, Italy), J. Ryu (Mayo Clinic, Rochester, NY, USA), H. Schunemann (McMaster University, Hamilton, Canada), M. Selman (Instituto Nacional de Enfermedades Respiratorias, Mexico City, Mexico), J. Swigris (National Jewish Health), A. Wells (Royal Brompton Hospital), and librarians R. Dudden, B. Griss and S. Protzko (all National Jewish Health).

I would also like to thank the primary sponsor, the American Thoracic Society (ATS), and the co-sponsors, the European Respiratory Society (ERS), the Japanese Respiratory Society (JRS) and the Asociación Latinoamericana de Tórax (ALAT) for supporting the development of the new guidelines for IPF. Finally, my thanks also go to J. Corn and K. Wilson (members of the documentation and implementation committee of the ATS (New York, NY, USA)) for providing useful input for this editorial.

\section{REFERENCES}

1 Idiopathic pulmonary fibrosis: diagnosis and treatment. International Consensus Statement. American Thoracic Society (ATS), and the European Respiratory Society (ERS). Am J Respir Crit Care Med 2000; 161: 646-664.

2 Raghu G, Collard HR, Egan JJ, et al. An Official ATS/ERS/JRS/ ALAT Statement: Idiopathic pulmonary fibrosis: evidence-based guidelines for diagnosis and management. Am J Respir Crit Care Med 2011; 183: (In press).

3 Schünemann HJ, Jaeschke R, Cook DJ, et al. An official ATS statement: grading the quality of evidence and strength of recommendations in ATS guidelines and recommendations. Am J Respir Crit Care Med 2006; 174: 605-614.

4 Raghu G. Improving the standard of care for patients with idiopathic pulmonary fibrosis requires participation in clinical trials. Chest 2009; 136: 330-333.

5 Vancheri C, Failla M, Crimi N, et al. Idiopathic pulmonary fibrosis: a disease with similarities and links to cancer biology. Eur Respir J 2010; 35: 496-504.

6 ATS patient information series: what is idiopathic pulmonary fibrosis? Am J Respir Crit Care Med 2011; (In press).

7 The IPFnet Strategy. Creating a comprehensive approach in the treatment of idiopathic pulmonary fibrosis. Am J Respir Crit Care Med 2010; 181: 527-528.

8 Guenther A, Eickelberg O, Preissner KT, et al. International registry for idiopathic pulmonary fibrosis. Thorax 2008; 63: 841.

9 Guenther A. The European IPF Network: towards better care for a dreadful disease. Eur Respir J 2011; 37: 744-772. 